\title{
Correction to: Switchable and Enhanced Absorption via Qubit-Mechanical Nonlinear Interaction in a Hybrid Optomechanical System
}

\author{
Amjad Sohail $^{1} \cdot$ Rizwan Ahmed $^{2} \cdot$ Chang shui $\mathrm{Yu}^{3}$
}

Published online: 8 February 2021

(C) Springer Science+Business Media, LLC, part of Springer Nature 2021

\section{Correction to: Int J Theor Phys \\ https://doi.org/10.1007/s10773-020-04655-2}

The author found some inconsistencies regarding the figures in the published version. The following details are the corrections that were rectified in the published original version of the article:

1) In the published version, Fig. 1 (b) is actually the image for Fig. 3. It must be placed after Fig. 2 with the caption labeled in "Fig. 3".

2) Fig. 2 in the published version is at right position with caption.

3) In the published version, the image in Fig. 3 is actually for Fig. 4. Thus, the image in Fig. 3 must be shifted to Fig. 4. However, the caption remains same.

4) Similarly, the image in Fig. 4 in the published version must be shifted to Fig. 5. However, the caption remains same.

5) Fig. 6 in published version is at right place with caption.

6) Figure 7 is actually Fig. 7(b) while the image for Fig. 7(a) (shown in the next page) was missing in the published version. However, the caption remains same.

The online version of the original article can be found at https://doi.org/10.1007/s10773-020-04655-2

Amjad Sohail

amjadsohail@gcuf.edu.pk

1 Department of Physics, Government College University, Allama Iqbal Road, Faisalabad 38000, Pakistan

2 Physics division, Pakistan institute of nuclear science and technology (PINSTECH), Nilore, Islamabad 45650, Pakistan

3 School of Physics, Dalian University of Technology, Dalian 116024, People's Republic of China 


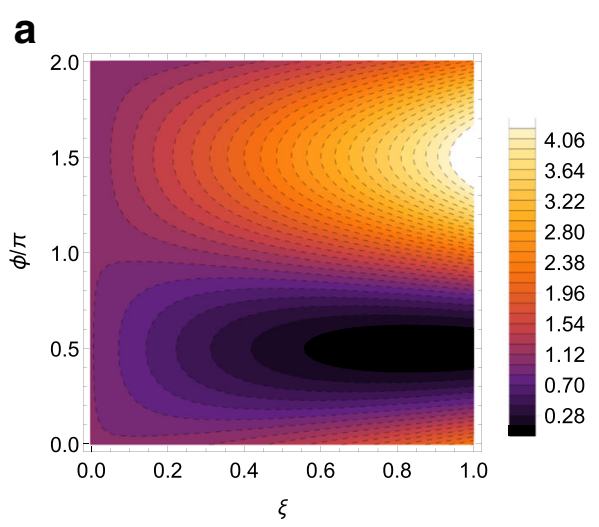

7) Figure 8 in published version is at right place with caption.

The original article has been corrected.

Publisher's Note Springer Nature remains neutral with regard to jurisdictional claims in published maps and institutional affiliations. 